\title{
Osteogenic potency of dedifferentiated fat cells isolated from elderly people with osteoporosis
}

\author{
WEI HAO $^{1 *}$, CHUANQIANG JIANG ${ }^{2 *}$, MING JIANG ${ }^{3 *}$, TIAN WANG ${ }^{4}$ and XIN WANG ${ }^{1}$ \\ ${ }^{1}$ Department of Orthopedics and Traumatology, Yantai Yuhuangding Hospital Affiliated to Qingdao University \\ Medical College; ${ }^{2}$ Department of Orthopedics and Traumatology, Yantaishan Hospital, Yantai, Shandong 264000; \\ ${ }^{3}$ Department of Stomatology, 107 Hospital of Jinan Military Area, Yantai, Shandong 264002; ${ }^{4}$ Department \\ of Pharmacology, School of Pharmacy, Yantai University, Yantai, Shandong 264005, P.R. China
}

Received August 27, 2015; Accepted January 26, 2017

DOI: $10.3892 /$ etm.2017.4465

\begin{abstract}
Mature adipocytes are the major cell type in adipose tissue. This study aimed to explore the osteogenic potency of dedifferentiated fat cells obtained from osteoporotic patients (opDFATs) in vitro and in vivo. Mature adipocytes and adipose-derived stem cells (opASCs) were harvested from subcutaneous adipose tissue. Mature adipocytes were dedifferentiated to produce opDFATs by the ceiling culture method. OpDFATs were osteogenically induced in vitro with opASCs as a control. Cell growth, alkaline phosphatase (ALPase) activity and cell mineralization were determined, and expression levels of osteogenesis-specific genes (collagen I, osteocalcin and bone sialoprotein) were analyzed using quantitative reverse transcription polymerase chain reaction. After 14 days, the opDFATs were combined with a poly(lactide-co-glycolide)$\beta$-tricalcium phosphate porous scaffold after being suspended in collagen I gel and implanted into nude mice for 4 weeks prior to histological analysis. Unilocular lipid droplets in mature adipocytes gradually split into smaller droplets and disappeared from the cytoplasm. Mature adipocytes dedifferentiated to opDFATs and cell morphology changed from spherical to elongated. High levels of ALPase and cell mineralization were observed in opDFATs by staining. No significant differences were found between the growth curves, ALPase activity, cell mineralization and expression levels of osteogenesis-specific genes between opDFATS and opASCs. After implantation for 4 weeks, new bone tissue was observed
\end{abstract}

Correspondence to: Dr Wei Hao or Dr Xin Wang, Department of Orthopedics and Traumatology, Yantai Yuhuangding Hospital Affiliated to Qingdao University Medical College, 20 Yuhuangding Road, Yantai, Shandong 264000, P.R. China

E-mail: ehaw@163.com

E-mail:newpule@163.com

${ }^{*}$ Contributed equally

Key words: mature adipocytes, osteogenesis, dedifferentiated fat cells histologically in the opDFATs-based biocomposite. OpDFATs are implicated as a novel type of seed cell for bone tissue engineering based on their osteogenic potency and higher abundance in adipose tissue compared with opASCs.

\section{Introduction}

In an aging population, osteoporosis, which is defined as bone mineral density reduction and bone microarchitecture deterioration, is becoming an increasingly common health problem (1). Even minor trauma, such as a fall from standing height, is sufficient to cause severe fractures at multiple sites (principally in the spine, hip, distal radius and proximal humerus (1). Such fractures are referred to as fragility fractures and are exclusively related to osteoporosis (1). Furthermore, once these fractures occur, measures adopted for osteoporosis prevention and treatment become difficult and less effective (1). If internal fixation techniques fail (due to screw loosening or pull-out), aseptic prosthetic loosening after arthroplasty and periprosthetic fractures become more likely due to decreased strength and increased fragility of the osteoporotic bone (1). This situation may be worsened when reconstructing large bone defects and non-unions become unavoidable after major trauma or tumor resections (1). To date, autologous cancellous bone grafting remains the therapeutic gold standard method used to reinforce bone mass and strength; however, limited graft availability, donor site morbidity and decreased bone marrow osteogenesis limit its use for this type of clinical application (2-4).

Cell-based bone tissue engineering holds great promise. Selecting suitable seed cells, which possess favorable bone formation capacity, is critical for the fabrication of eligible bioengineering composites. The osteogenic potency of bone marrow mesenchymal stem cells (BMSCs) and adipose tissue mesenchymal stem cells (ASCs) has been widely identified and used to enhance bone tissue formation by combination with bioengineering scaffolds and/or osteogenic cytokines $(1,5,6)$.

However, widespread application of BMSCs and ASCs for this purpose is unsuitable in elderly patients. Osteoporosis is frequently related to obesity $(7,8)$, although the underlying mechanism for this has not yet been fully elucidated. Osteoblasts 
and adipocytes are both derived from BMSCs; therefore, decreased osteogenesis and increased adipogenesis of BMSCs increases bone marrow adiposity and also enhances body fat deposition $(9,10)$. The application of BMSCs with reduced osteogenic capacity could limit the potential of these cells for the treatment of bone defects. However, abundant fat tissues could be a source of alternatives to BMSCs for this purpose. Compared with BMSCs, ASCs exhibit superior osteogenic capacity, and could be used to maintain adequate capability in elderly people suffering from osteoporosis $(1,11,12)$. As terminally differentiated cells, mature adipocytes can be transformed into more primitive dedifferentiated fat cells (DFATs), which regain multilineage differentiation potential (13-15). After osteogenic differentiation, DFATs combined with biocomposites have been used to form new bone tissue in ectopic sites and to repair lacunar bone defects $(14,16)$. Derived from mature adipocytes, which constitute the major part of adipose tissue, DFATs are a more abundant cell type compared with ASCs. Therefore, DFATs should be a key focus of bone tissue engineering research, especially for the treatment of osteoporosis and related diseases.

Previous studies have demonstrated that ASCs from osteoporotic patients (opASCs) possess favorable osteogenic potency (1). In the present study, DFATs were derived from the fat cells of osteoporotic patients (opDFATs) and their osteogenic potential was evaluated both in vitro and in vivo.

\section{Materials and methods}

Isolation and culture of opDFATs and opASCs. Before cell harvesting, related protocols were approved by the Medical Ethics Committee of Yantai Yuhuangding Hospital Affiliated to Qingdao University Medical College (Yantai, China; approval no. YYYLLS[2014]126) and written informed consent was provided by all patients. Subcutaneous fat tissues were obtained from 12 patients between January and December 2014 ( 8 females and 4 males; average age $65.2 \pm 16.8$ years) during surgery for the repair of hip fractures by internal fixation or total hip arthroplasty. The adipose tissues were washed with sterilized phosphate buffered saline, finely minced and digested with $0.1 \%$ collagenase I (Sigma-Aldrich; Merck KGaA, Darmstadt, Germany) at $37^{\circ} \mathrm{C}$ for $1 \mathrm{~h}$. Collagenase I was neutralized by adding an equal volume of control medium containing Dulbecco's modified Eagle's medium (Gibco; Thermo Fisher Scientific, Inc.) and $10 \%$ fetal bovine serum (Hangzhou Sijiqing Biological Engineering Materials Co., Ltd., Hangzhou, China), then the tissues were filtered through a $150-\mu \mathrm{m}$ mesh filter to remove the debris. The filtrate was centrifuged at $150 \mathrm{x} \mathrm{g}$ for $6 \mathrm{~min}$. The top layer, containing unilocular adipocytes, and the pellet were collected separately. The pellet was resuspended and processed consecutively to isolate and culture opASCs in vitro as previously described (1).

Mature adipocytes were isolated using the ceiling culture method. A total of $\sim 5 \times 10^{4}$ mature adipocytes were placed in each $25 \mathrm{~cm}^{2}$ culture flask (Nalge Nunc International, Penfield, NY, USA) that were completely filled with the control medium and incubated at $37^{\circ} \mathrm{C}$ under $5 \% \mathrm{CO}_{2}$. The cells floated and adhered to the top inner layer of the flasks. During the first 3-4 days, mature adipocytes adhered loosely to the ceiling surface and cell movement could be observed with gentle shaking of flasks. At day 7-8, the cells started to lose their spherical shapes and adhered more strongly to the ceiling layer; no cell movement could be detected with shaking. After sufficient attachment of cells (usually 10-12 days), the flask was reinverted. The medium was removed and replaced every 3 days with $\sim 5 \mathrm{ml}$ on each occasion. At confluence, the cells were passaged with $0.25 \%$ trypsin/EDTA and replated at a 1:3 dilution.

In vitro osteogenic differentiation. OpDFATs and opASCs at passage 2 were induced in 6 -well plates $\left(2 \times 10^{4}\right.$ cells/well $)$ in osteogenic differentiation medium [control medium supplemented with $0.1 \mu \mathrm{M}$ dexamethasone, $50 \mu \mathrm{g} / \mathrm{ml}$ ascorbic acid-2-phosphate and $10 \mathrm{mM} \beta$-glycerophosphate (all Sigma-Aldrich; Merck KGaA)]. The medium was replaced every 3 days. In order to evaluate the osteogenic potency of opDFATs, alkaline phosphatase (ALPase) activity and calcium deposition assays were conducted using the diazo coupling method and von Kossa staining as previously described $(17,18)$, respectively, at 14 and 21 days after induction.

The DNA content of opDFAT and opASC wells was determined by fluorometric assay. At day 1, 7 and 14, cells were treated with trypsin, collected by centrifugation at $150 \mathrm{x} \mathrm{g}$ for $6 \mathrm{~min}$ at room temperature and lysed by sonication. The homogenate was mixed with Hoechst 33258 stain (Dojindo Molecular Technologies, Inc., Kumamoto, Japan). Emission and excitation spectra were obtained using a Modulus Microplate Luminometer (Turner BioSystems; Promega Corporation, Madison, WI, USA) at 458 and $356 \mathrm{~nm}$, respectively.

ALPase activity and cell mineralization measurement under osteogenic differentiation in vitro. At day 7 and 14, opDFATs and opASCs were lysed by trypsinization, centrifugation and sonication. The ALPase activity of cell lysates was determined by measuring the release of $\mathrm{p}$-nitrophenol from $\mathrm{p}$-nitrophenyl phosphate (Sigma-Aldrich; Merck KGaA). The release of p-nitrophenol was monitored by measuring the optical density at $405 \mathrm{~nm}$. The optical densities were then compared with a standard p-nitrophenol solution (Sigma-Aldrich; Merck $\mathrm{KGaA}$ ). The protein concentrations of cell lysates were determined biochemically using the Bicinchoninic Acid Protein Assay kit (Pierce; Thermo Fisher Scientific, Inc.) according to the manufacturer's protocols. Results are expressed as nmol p-nitrophenol per $\mu \mathrm{g}$ protein per min.

For evaluation of cell mineralization, cell lysates were obtained using the aforementioned method and incubated overnight at $4^{\circ} \mathrm{C}$ with $1 \mathrm{ml} 0.5 \mathrm{~N} \mathrm{HCl}$ with gentle shaking. $\mathrm{Ca}^{2+}$ ion levels were determined using the o-cresolphthalein complexone method with a commercial Calcium C kit (Wako Pure Chemical Industries, Ltd., Osaka, Japan) according to the manufacturer's protocols.

Reverse transcription-quantitative polymerase chain reaction (RT-qPCR) of osteogenesis-specific genes under osteogenic differentiation in vitro. At days 1, 7 and 14, total RNA was extracted from opDFATs and opASCs using TRIzol reagent (Invitrogen; Thermo Fisher Scientific, Inc.) and was reverse-transcribed to cDNA using a PrimeScript ${ }^{\mathrm{TM}}$ RT reagent kit (Takara Biotechnology Co., Ltd., Dalian, China) according to the manufacturer's protocol. Then, qPCR assays 
were performed in a total volume of $25 \mu \mathrm{l}$ containing $1 \mu \mathrm{l}$ cDNA, $10 \mu \mathrm{M}$ gene-specific primers, 2x SYBR Premix Ex Taq $^{\mathrm{TM}}$ (Takara Biotechnology Co., Ltd.), 50x ROX Reference Dye and $\mathrm{dH}_{2} \mathrm{O}$, in an ABI 7500 Real-Time Thermocycler (Applied Biosystems; Thermo Fisher Scientific, Inc.). The reaction conditions were as follows: Initial denaturation at $94^{\circ} \mathrm{C}$ for $10 \mathrm{~min}$, followed by 45 cycles of $94^{\circ} \mathrm{C}$ for $40 \mathrm{sec}, 60^{\circ} \mathrm{C}$ for $30 \mathrm{sec}$ and $72^{\circ} \mathrm{C}$ for $30 \mathrm{sec}$. $\beta$-actin was used as an internal control to evaluate total RNA input. The expression levels of the osteogenesis-specific genes collagen I (COL I), osteocalcin (OC) and bone sialoprotein (BSP) were calculated using the comparative threshold-cycle method. The efficiency of each assay was calculated using the formula $E=10^{-1 / \text { slope }}$. The following primer sequences were used in RT-qPCR assays (sense, antisense): $\beta$-actin (5'-ACAGAGCCTCGCCTTTGCC-3', 5'-ACATGCCGGAGCCGTTGTC-3'), COL I (5'-GCAAGGG AGAAAAGGGTGAACC-3', 5'-GTGGCTCCAGCAGGAC CAG-3'), OC (5'-CTCCAGGCACCCTTCTTTCC-3', 5'-ATTC CTCTTCTGGAGTTTATTTGGG-3'), BSP (5'-ATACCATCT CACACCAGTTAGAATG-3', 5'-AACAGCGTAAAAGTGT T CCTAT TTC-3').

In vivo implantation of opDFATs. Approval was obtained from the Institutional Animal Review Committee of Yantai Yuhuangding Hospital Affiliated to Qingdao University Medical College (approval no. YYYDWS[2014]058) prior to beginning animal research in this study. A total of 6 4-week-old nude mice (sex ratio 1:1; body weight 14-16 g) were bought from the Animal Center of BinZhou Medical College (Yantai, China) and housed in specific pathogen free cages, supplied with autoclaved food, water and bedding ad libitum at $26-28^{\circ} \mathrm{C}$ with $40-60 \%$ humidity at the animal center of BinZhou Medical College, (Yantai, China). Before implantation, collagen I gel and poly (lactide-co-glycolide) $/ \beta$ -tricalcium phosphate (PLGA- $\beta$-TCP) porous scaffold were prepared as previously described (1). Passage 2 opDFATs were suspended in $100 \mu \mathrm{l}$ collagen I at $2 \times 10^{6}$ cells $/ \mathrm{ml}$ and seeded into the porous PLGA- $\beta$-TCP scaffold on ice. They were then incubated at $37^{\circ} \mathrm{C}$ for $30 \mathrm{~min}$ to allow gel formation to fabricate the opDFAT-COL/PLGA- $\beta$-TCP composite. The constructs were cultured in osteogenic medium at $37^{\circ} \mathrm{C}$ in an atmosphere containing $5 \% \mathrm{CO}_{2}$ for 14 days. Mice were anesthetized with $1 \%$ pentobarbital sodium at $40 \mathrm{mg} / \mathrm{kg}$ (Sigma-Aldrich; Merck KGaA) and constructs were implanted into the space between the subcutaneous tissue and the deep fascia of nude mice. The mice were sacrificed by cervical dislocation 4 weeks later and the implants were harvested and prepared for histological analyses by hematoxylin and eosin staining. An acellular COL/PLGA- $\beta$-TCP composite was implanted as a blank control.

In addition, scanning electron microscopy (SEM) was used to observe cell adhesion and fabrication structure of the opDFAT-COL/PLGA- $\beta$-TCP composite. Briefly, the osteogenic medium was removed following 14 days of in vitro culture and the cell-scaffold composites were washed 3 times with PBS, fixed with $2.5 \%$ glutaraldehyde, dehydrated through a graded series of ethanol, and critical point dried. Samples were fixed onto SEM aluminum stubs, sputter coated with gold and observed under a scanning electron microscope (Hitachi Ltd., Tokyo, Japan).
Statistical analysis. All quantitative data are expressed as the mean \pm standard deviation. SPSS 19.0 software (IBM SPSS, Armonk, NY, USA) was used for data analysis. Student's t-tests were used to analyze the results of ALPase activity and extracellular mineralization assays. The relative expression level of osteogenesis-specific genes was compared by analysis of variance. $\mathrm{P}<0.05$ was considered to indicate a statistically significant difference.

\section{Results}

In vitro osteogenic differentiation of opDFATs. At day 1 , the unilocular mature adipocytes floated on the top layer of the medium and made contact with the inner ceiling surface of the flasks (Fig. 1A). Small movements of the mature adipocytes were observed with gentle shaking. At day 6-7, the unilocular adipocytes adhered tightly to the inner ceiling surface of the flasks. Mature adipocytes started to lose their spherical shape and exhibit fibroblast-like morphology. Unilocular lipid droplets in the cytoplasm gradually split into smaller droplets at day 10-12 (Fig. 1B). The culture medium was then replaced and the flasks were reinverted so that the cells were cultured on the bottom surface. Primary culture duration to reach confluence was usually 15-20 days (Fig. 1C).

Subsequently, the cells were passaged with $0.25 \%$ trypsin/EDTA and replated at a 1:3 dilution. The primary culture of opASCs was performed as described previously (8). OpDFATs and opASCs at passage 2 were cultured in osteogenic medium and received further examination. As shown in Fig. 1D, opDFATs and opASCs exhibited similar growth profiles. The DNA content of opDFATs increased continuously, with no statistically significant differences detected as compared with opASCs.

ALPase activity and extracellular mineralization of opDFATs. From day 7 to 14 , the ALPase activity of opDFATs was found to be significantly enhanced ( $\mathrm{P}<0.05$; Fig. 2A). There was no statistically significant difference between ALPase activity levels in opDFATs and opASCs (Fig. 2A). The results of the diazo coupling method revealed an extensive purple-stained area, confirming ALPase activity in opDFATs (Fig. 2B).

Extracellular mineralization of opDFATs increased significantly from day 7 to 14 ( $\mathrm{P}<0.05$; Fig. 2C). No statistically significant difference was detected between extracellular mineralization levels in opDFATs and opASCs (Fig. 2C). Von Kossa staining of extracellular matrix mineralization confirmed that calcium deposition was present in opDFAT cell culture, visible as black nodules (Fig. 2D).

Osteogenesis-specific gene expression. COL I expression in opDFATs increased significantly at days 4, 7 and 14 as compared with day 1 (all $\mathrm{P}<0.05$; Fig. 3A), with no significant differences in COL I expression levels observed between opDFATs and opASCs. Compared with day 1, a continuous increase in OC expression was observed in opDFATs on days 4, 7 and 14, with significant differences at each time point (all $\mathrm{P}<0.05$; Fig. 3B). No significant differences in OC expression levels were observed between opDFATs and opASCs. Similarly, BSP expression in opDFATs increased continuously 

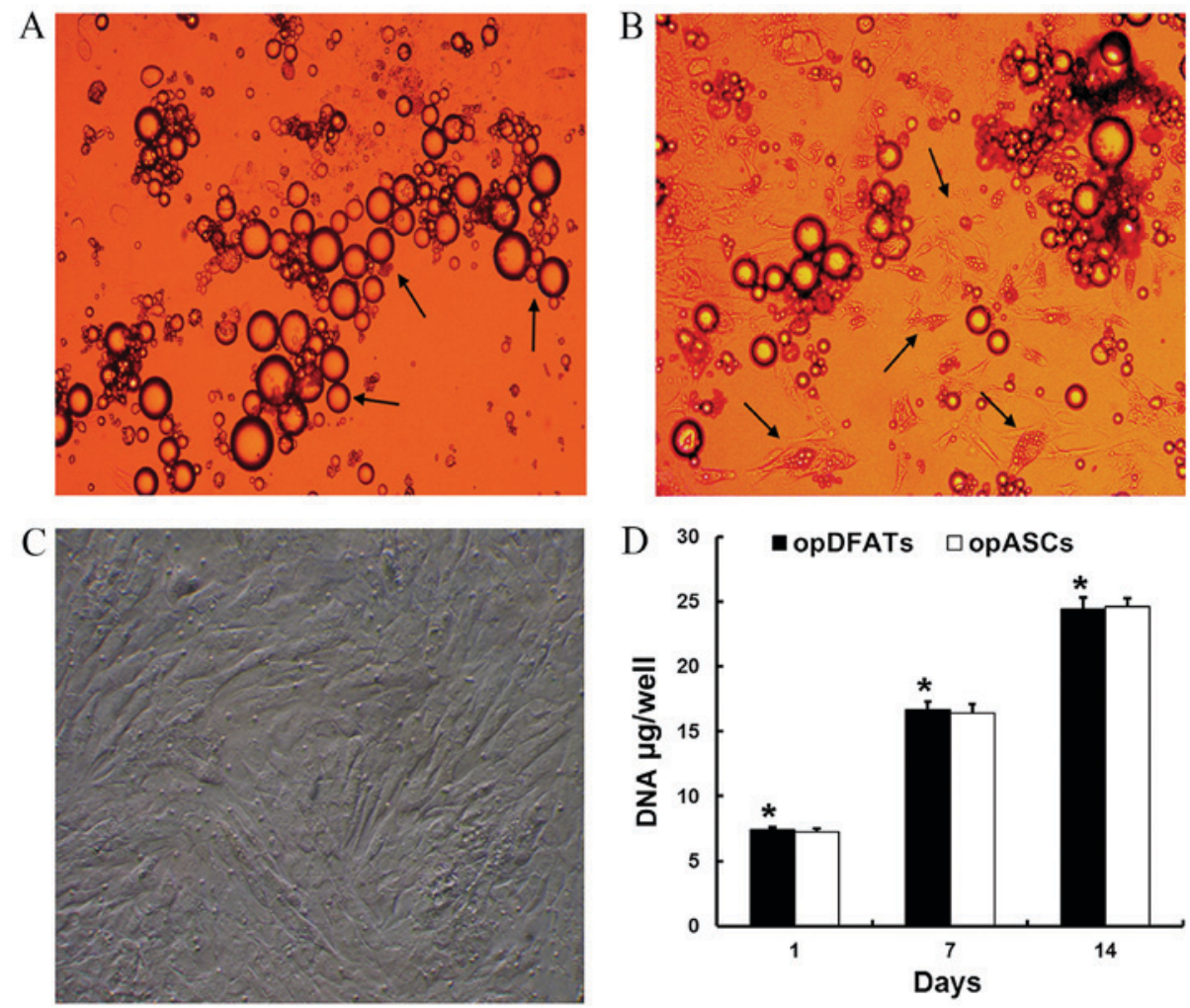

Figure 1. In vitro culture of opDFATs. (A) Mature adipocytes obtained using the ceiling culture method. Black arrows indicate unilocular lipid droplets (B) Mature adipocyte morphology changed from spherical to elongated. Black arrows indicate unilocular lipid droplets that have split into smaller droplets. (C) Mature adipocytes dedifferentiated into fibroblast-like opDFATs and reached confluence. (D) DNA content of opDFATs compared with opASCs under osteogenic induction. Magnification, $\mathrm{x} 20 .{ }^{*} \mathrm{P}<0.05$, statistically significant difference between opDFATs at different culture time-points. opDFAT, dedifferentiated fat cell obtained from an osteoporotic patient; opASC, adipose-derived stem cell obtained from an osteoporotic patient.
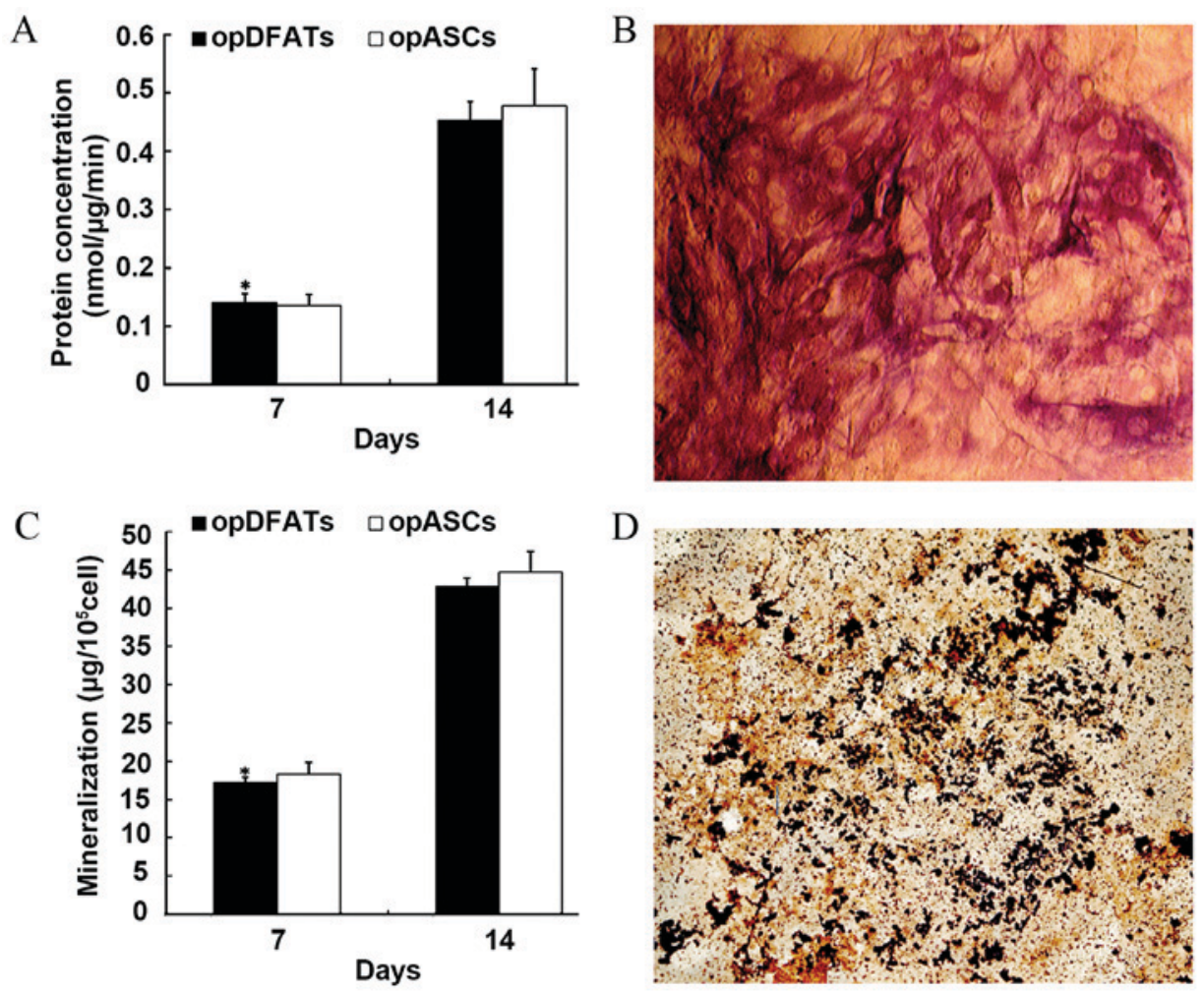

Figure 2. (A and B) ALPase activity and (C and D) extracellular mineralization of opDFATs. Quantitative expression of (A) ALPase activity (C) extracellular mineralization in opDFATs. Qualitative staining of (B) ALPase activity and (D) extracellular mineralization in opDFATs after osteogenic induction for 14 and 21 days respectively. Expression of ALPase activity can be observed as purple-stained area and calcium deposition as black nodules. Magnification, x20. "P<0.05 vs. day 14 , statistically significant difference between opDFATs. ALPase; alkaline phosphatase opDFAT, dedifferentiated fat cell obtained from an osteoporotic patient; opASC, adipose-derived stem cell obtained from an osteoporotic patient. 

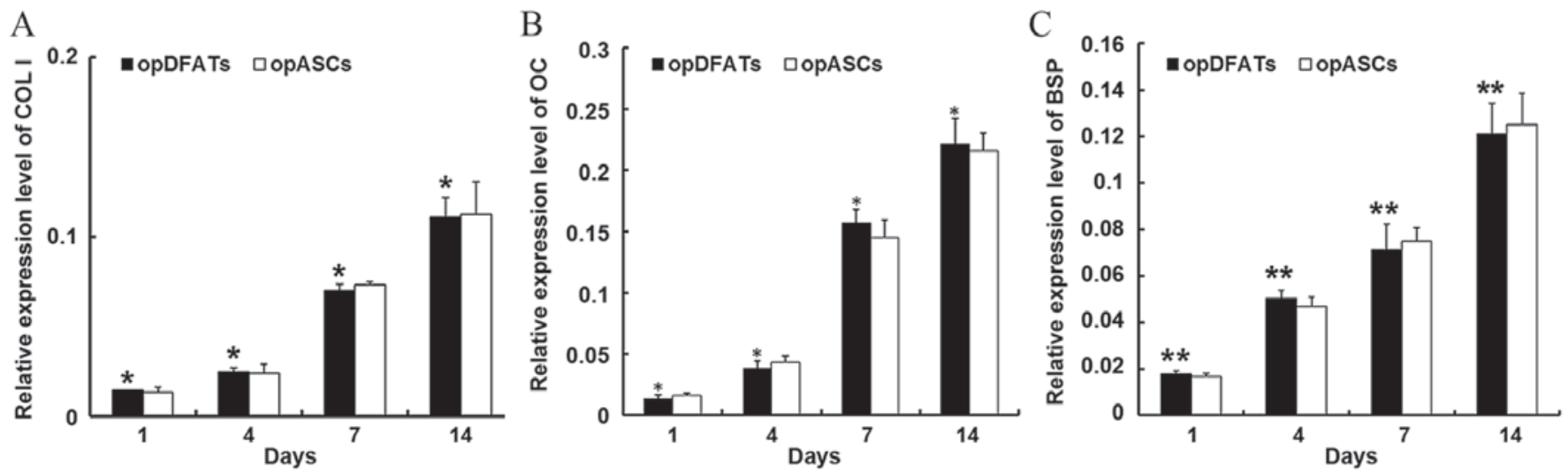

Figure 3. Osteogenesis-specific gene expression of opDFATs and opASCs under osteogenic induction. Reverse transcription-quantitative polymerase chain reaction was conducted to evaluate (A) COL I, (B) OC and (C) BSP expression levels. " $\mathrm{P}<0.05$, statistically significant difference among opDFATs at different culture time-points (COL I and OC); ${ }^{* *} \mathrm{P}<0.05$, statistically significant difference between opDFATs at days $1,4,7$ and 14 (BSP). COL I, collagen I; OC, osteocalcin; BSP, bone sialoprotein; opDFAT, dedifferentiated fat cell obtained from an osteoporotic patient; opASC, adipose-derived stem cell obtained from an osteoporotic patient.
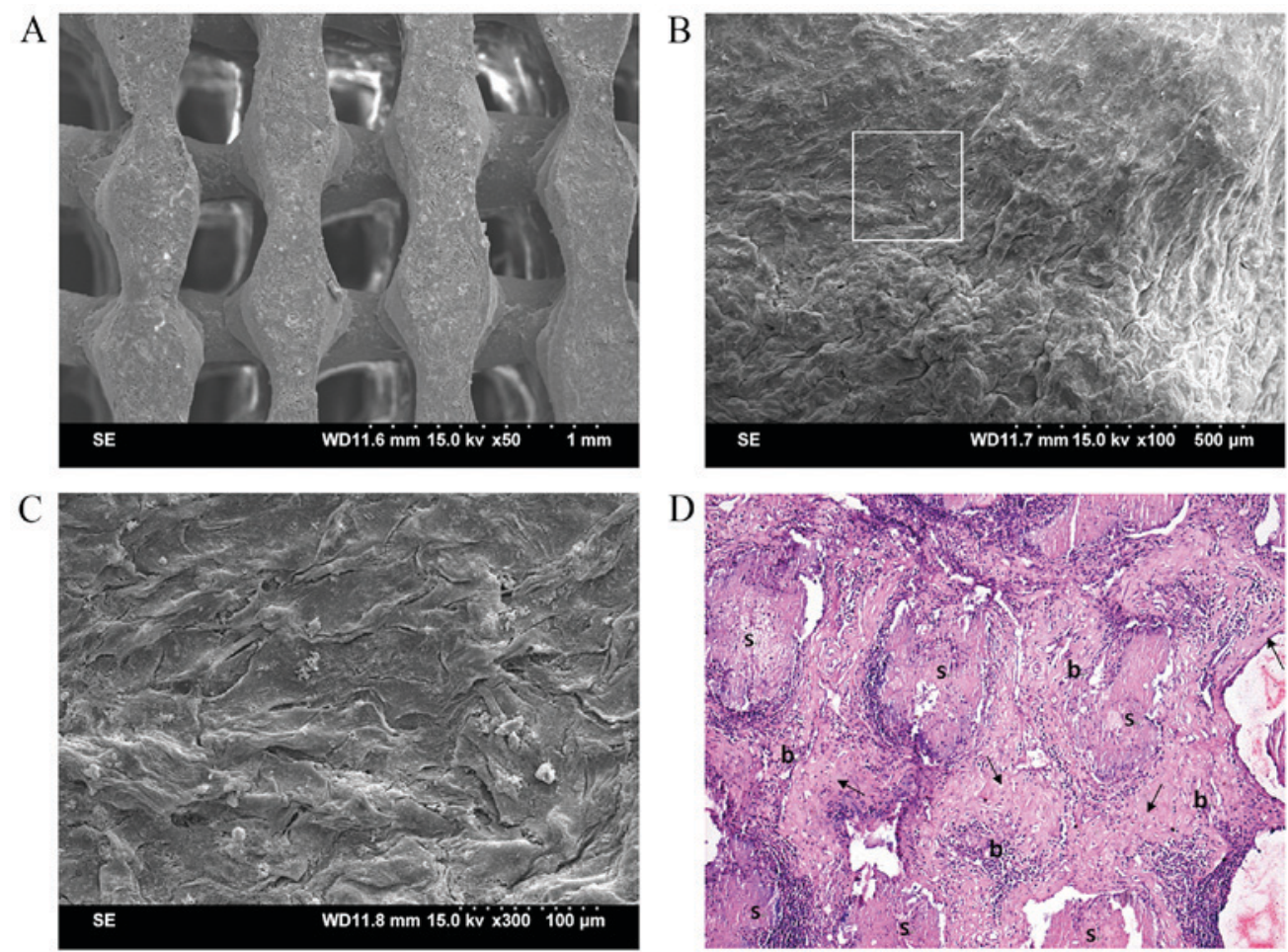

Figure 4. In vivo implantation of an opDFAT-based composite. Scanning electron microscope images of (A) PLGA- $\beta$-TCP scaffold (magnification, $x 100)$ and (B) opDFAT-COL/PLGA- $\beta$-TCP (magnification, x100). (C) Magnified view of the insert from B, in which opDFATs can be seen in close contact (magnification, x200). (D) Hematoxylin and eosin staining. Black arrows indicate typical osteocytes, 's' indicates scaffold and 'b' indicates bone tissue. Magnification, x100. opDFAT, dedifferentiated fat cell obtained from an osteoporotic patient; opASC, adipose-derived stem cell obtained from an osteoporotic patient.

from day 1 to days 4,7 and 14 , with significant differences between each time point (all $\mathrm{P}<0.05$; Fig. $3 \mathrm{C}$ ). No significant differences in BSP expression levels were observed between opDFATs and opASCs.

In vivo implantation of the opDFATs-based composite. For the opDFAT-COL/PLGA- $\beta$-TCP composite, surfaces and pores of the PLGA- $\beta$-TCP scaffold (Fig. 4A) were filled with opDFATs, which were entrapped by collagen I gel as demonstrated by SEM (Fig. 4B and C). Four weeks after in vivo implantation, woven bone tissue with visible osteocytes containing lacunae was formed evenly in the pores of the opDFAT-COL/PLGA- $\beta$-TCP composite (Fig. 4D). In contrast, no bone tissue was observed except connective tissues in the COL/PLGA- $\beta$-TCP composite (data not shown).

\section{Discussion}

In the present study, it was demonstrated that mature adipocyte-derived DFATs isolated from subcutaneous fat tissues of osteoporotic patients (opDFATs) possess osteogenic differentiation capacity equivalent to that of opASCs. 
Treatment of osteoporotic fractures is highly demanding because of the inherent changes in bone tissue, including reduced bone mineral density, unfavorable geometry at cortical bone sites and deteriorated bone microarchitecture $(2,3)$. Cell-based tissue engineering can be used to augment bone mass, strengthen osteointegration around the periprosthetic region and reconstruct bone defects. BMSCs exhibit a low proliferative capacity and decreased osteogenic potential among the elderly population, especially those individuals with osteoporosis $(19,20)$. Harvesting and in vitro culture of BMSCs obtained from this population is problematic due to increased patient distress, donor site morbidity, infection risk and poor osteogenic capacity (1). These problems decrease the feasibility of translating the isolation of BMSCs from osteoporotic patients into a potentially clinical application (1).

Previous results have suggested that osteoporosis and obesity share common etiological features. These include overlapping genetic and environmental predisposition, high concurrence of osteoporosis and bone marrow adiposity during normal aging, similar regulation mechanisms via the hypothalamus and sympathetic nervous system for bone remodeling and adiposity, and common progenitor mesenchymal stem cells (7). However, the interaction between fat mass and bone is complex. Previous studies have indicated that excessive fat mass is associated with beneficial effects on bone that protect against osteoporosis $(21,22)$, although conflicting results have also been reported $(23,24)$. This discrepancy may be due to complicated regulation mechanisms, which are mediated by adipocyte-derived peptides, pancreatic hormones and mesenchymal stem cell differentiation (25).

In relation to bone tissue engineering, excessive fat mass is favorable because it represents an abundant seed cell source that can be utilized to treat osteoporosis-related fractures (1). Adipose tissue contains adipocytes and non-adipose cells (ASCs, fibroblasts, endothelial cells, blood cells and macrophages) (26). Studies conducted both in vitro and in vivo have demonstrated that human ASCs can differentiate towards multiple lineages, such as adipocytes, osteoblasts, chondrocytes, myocytes, neuronal cells, endothelial cells and hepatocytes (27). ASCs have been demonstrated to maintain proliferative and osteogenic differentiation capacity, particularly in elderly people with osteoporosis $(1,11,12)$; however, ASCs account for only a small proportion of the total cells in adipose tissue. Several studies have also demonstrated that ASCs are a heterogeneous population containing smooth muscle cells, endothelial cells, mast cells and lineage-committed progenitor cells $(28,29)$. These factors could hinder the osteogenic induction process of ASCs, by making in vitro culturing more difficult and time-consuming before implantation in vivo.

Mature adipocytes are functionally the most important cell type in adipose tissue, with a typical morphology characterized by the presence of a single, large cytoplasmic lipid droplet accounting for $\sim 90 \%$ of its volume (26). As demonstrated in this study, mature adipocytes can be isolated using the traditional ceiling culture method. The unilocular lipid droplets in mature adipocytes cause the cells to float in the culture medium and they can therefore be easily isolated from other cell populations. Previously, flow cytometric analysis has revealed that a highly homogeneous population of opDFATs cells can be obtained using this technique (26). As terminally differentiated cells, mature adipocytes can be dedifferentiated and transdifferentiated towards multiple lineages, including chondrocytes, osteoblasts, skeletal myocytes, smooth muscle cells and cardiomyocytes (26,30-33). During ceiling culture in vitro, it was observed that the lipid droplets in mature adipocytes gradually diminished, accompanied by changes in cell morphology from spherical to elongated. The cells then dedifferentiated as opDFATs and entered a proliferation phase similar to opASCs. In vitro investigations suggested that opDFATs possess favorable osteogenic potential comparable to that of opASCs. Furthermore, in vivo studies confirmed ectopic bone tissue formation of opDFAT-based bioengineering composites. Previous studies have demonstrated the osteogenic capacity of DFATs and related biocomposites (14,34); however, there have been few reports of osteogenesis-related research on DFATs isolated from osteoporotic patients. DFATs harvested from the ovariectomy-induced osteoporosis model in rabbits showed osteogenic activity similar to cells from healthy samples (16). Intra-bone marrow injection of autologous DFAT cells significantly increased the bone mineral density at the injected site (16). Hence, opDFATs could become an alternative to opASCs as a seed cell source for bone defect repair in osteoporotic patients by bone tissue engineering methods.

Previous results of molecular analysis indicate that mature adipocytes contain transcripts for embryonic stem cell genes, which are required for self-renewal and pluripotency (15). During the dedifferentiation process in culture, DFATs lose mature adipocyte markers including lipoprotein lipase, leptin, glucose transporter type 4 , adiponectin, adipocyte protein 2 and preadipocyte factor- $1(15,26)$. Transcription factors which regulate adipocytic differentiation (peroxisome proliferator-activated receptor gamma and CCAAT-enhancer-binding proteins $\alpha, \beta$ and $\delta$ ) are also significantly downregulated, while those that are critical for osteogenesis and chondrogenesis (runt-related transcription factor 2 and SRY-box 9) are expressed during dedifferentiation (26). Flow cytometric analysis demonstrated that DFATs exhibit uniform cell surface protein expression similar to that of BMSCs (26). After dedifferentiation in culture, DFATs showed the capacity to transdifferentiate toward the osteogenic lineage under osteogenic induction in culture (34). Similar to BMSCs and ASCs, the BMP signaling pathways participate in this process. Upregulation of BMPR-IB has been shown to promote osteoblast differentiation of DFATs $(34,35)$.

In addition to the aforementioned confirmation of opDFATs as a suitable source of seed cells, it has previously been demonstrated that three-dimensional porous scaffolds act as micro frames for seed cells to adhere, proliferate and differentiate. PLGA- $\beta$-TCP (7:3 w/w) scaffolds with $90 \%$ porosity and 300 to $350 \mu \mathrm{m}$ pores were designed and produced using the low-temperature deposition manufacturing technique (36). Previous experiments by the current authors have demonstrated that the PLGA- $\beta$-TCP scaffold possesses good biocompatibility, biodegradability, osteoconductivity and mechanical properties after combination with ASCs in vitro and in vivo (5). In the present study, opDFATs were encapsulated in collagen I hydrogel and then combined with a PLGA- $\beta$-TCP porous scaffold. Collagen I hydrogel can hold large numbers of seed cells in porous scaffolds and promote their osteogenic 
differentiation $(5,12)$. SEM evaluation indicated that opDFATs were in close cell-to-cell contact, mediated via crosslinking of collagen I fibers and complete overgrowth around and into the porous scaffold. New bone tissue was formed after implantation of opDFAT-COL/PLGA- $\beta$-TCP composites in vivo.

Previous results have demonstrated that opASCs exhibit sustained proliferation and adequate osteogenicity in contrast to the impaired BMSCs in osteoporotic patients (8). In the present study, mature adipocytes were harvested and dedifferentiated to generate opDFATs. In vitro and in vivo osteogenic differentiation analyses suggested that opDFATs also exhibit favorable osteogenesis capacity. By using collagen I hydrogel as a cell carrier and incorporation with a PLGA- $\beta$-TCP scaffold, an opDFAT-COL/PLGA- $\beta$-TCP composite was fabricated and demonstrated to form new bone tissue in vivo. Future studies will focus on the repair of bone defects in orthotopic sites.

The present study is not without limitations. The osteogenic potential of opDFATs was primarily evaluated through in vitro culture and in ectopic sites with COL/PLGA- $\beta$-TCP scaffolds as cell carriers. Future studies should focus on a combination of opDFATs at different densities with various biomaterials and their bone formation capacity both in vitro and in vivo. The opDFATs-based biocomposites should be applied to repair larger bone defects in animals so as to provide more convincing data concerning sustained osteogenesis of opDFATs.

\section{Acknowledgements}

The authors acknowledge funding support from the Shandong Provincial Natural Science Foundation, China (grant no. ZR2012CQ018).

\section{References}

1. Jiang M, Wang X, Liu H, Zhou L, Jiang T, Zhou H and Hao W: Bone formation in adipose-derived stem cells isolated from elderly patients with osteoporosis: A preliminary study. Cell Biol Int 38: 97-105, 2014

2. Hao W, Hu YY, Wei YY, Pang L, Lv R, Bai JP, Xiong Z and Jiang M: Collagen I gel can facilitate homogenous bone formation of adipose-derived stem cells in PLGA-beta-TCP scaffold. Cells Tissues Organs 187: 89-102, 2008.

3. Lee JH, Lee JH, Park JW and Shin YH: The insertional torque of a pedicle screw has a positive correlation with bone mineral density in posterior lumbar pedicle screw fixation. J Bone Joint Surg Br 94: 93-97, 2012.

4. Wu ZX, Gong FT, Liu L Ma ZS, Zhang Y, Zhao X, Yang M, Lei $\mathrm{W}$ and Sang HX: A comparative study on screw loosening in osteoporotic lumbar spine fusion between expandable and conventional pedicle screws. Arch Orthop Trauma Surg 132: 471-476, 2012.

5. Hao W, Pang L, Jiang M, Lv R, Xiong Z and Hu YY: Skeletal repair in rabbits using a novel biomimetic composite based on adipose-derived stem cells encapsulated in collagen I gel with PLGA-beta-TCP scaffold. J Orthop Res 28: 252-257, 2010.

6. Hao W, Dong J, Jiang M, Wu J, Cui F and Zhou D: Enhanced bone formation in large segmental radial defects by combining adipose-derived stem cells expressing bone morphogenetic protein 2 with $\mathrm{nHA} / \mathrm{RHLC} / \mathrm{PLA}$ scaffold. Int Orthop 34: 1341-1349, 2010.

7. Rosen CJ and Bouxsein ML: Mechanisms of disease: Is osteoporosis the obesity of bone? Nat Clin Pract Rheumatol 2: 35-43, 2006.

8. Hagey AR and Warren MP: Role of exercise and nutrition in menopause. Clin Obstet Gynecol 51: 627-641, 2008.

9. Dalle Carbonare L, Valenti MT, Zanatta M, Donatelli L and Lo Cascio V: Circulating mesenchymal stem cells with abnormal osteogenic differentiation in patients with osteoporosis. Arthritis Rheum 60: 3356-3365, 2009.
10. Chen JR, Lazarenko OP, Wu X, Tong Y, Blackburn ML, Shankar K, Badger TM and Ronis MJ: Obesity reduces bone density associated with activation of PPAR $\gamma$ and suppression of Wnt $/ \beta$-catenin in rapidly growing male rats. PLoS One 5: e13704, 2010.

11. Chen HT, Lee MJ, Chen CH, Chuang SC, Chang LF, Ho ML, Hung SH, Fu YC, Wang YH, Wang HI, et al: Proliferation and differentiation potential of human adipose-derived mesenchymal stem cells isolated from elderly patients with osteoporotic fractures. J Cell Mol Med 16: 582-593, 2012.

12. Wu W, Niklason L and Steinbacher DM: The effect of age on human adipose-derived stem cells. Plast Reconstr Surg 131: 27-37, 2013.

13. Park SR, Oreffo RO and Triffitt JT: Interconversion potential of cloned human marrow adipocytes in vitro. Bone 24: 549-554, 1999.

14. Justesen J, Pedersen SB, Stenderup K and Kassem M: Subcutaneous adipocytes can differentiate into bone-forming cells in vitro and in vivo. Tissue Eng 10: 381-391, 2004.

15. Poloni A, Maurizi G, Leoni P, Serrani F, Mancini S, Frontini A, Zingaretti MC, Siquini W, Sarzani R and Cinti S: Human dedifferentiated adipocytes show similar properties to bone marrow-derived mesenchymal stem cells. Stem Cells 30: 965-974, 2012.

16. Kikuta S, Tanaka N, Kazama T, Kazama M, Kano K, Ryu J, Tokuhashi Y and Matsumoto T: Osteogenic effects of dedifferentiated fat cell transplantation in rabbit models of bone defect and ovariectomy-induced osteoporosis. Tissue Eng Part A 19: 1792-1802, 2013.

17. Hodson AW and Skillen AW. Comparison of diazo-coupling, formazan, and silver staining techniques for visualizing alkaline phosphatase isoenzymes after electrophoresis in homogeneous-pore and gradient-pore polyacrylamide gels. Anal Biochem 169:253-261, 1988.

18. Mallory FB: Pathological techniques: A practical manual for workers in pathological histology including directions for the performance of autopsies and for microphotography. WB Saunders, Philadelphia, PA, pp143-144, 1983.

19. Stolzing A, Jones E, McGonagle D and Scutt A: Age-related changes in human bone marrow-derived mesenchymal stem cells: Consequences for cell therapies. Mech Ageing Dev 129: 163-173, 2008.

20. Zhou S, Greenberger JS, Epperly MW, Goff JP, Adler C, Leboff MS and Glowacki J: Age-related intrinsic changes in human bone-marrow-derived mesenchymal stem cells and their differentiation to osteoblasts. Aging cell 7: 335-343, 2008.

21. Riis BJ, Rødbro P and Christiansen C: The role of serum concentrations of sex steroids and bone turnover in the development and occurrence of postmenopausal osteoporosis. Calcif Tissue Int 38: 318-322, 1986.

22. Lau EM, Chan YH, Chan M, Woo J, Griffith J, Chan HH and Leung PC: Vertebral deformity in chinese men: Prevalence, risk factors, bone mineral density, and body composition measurements. Calcif Tissue Int 66: 47-52, 2000.

23. Hsu YH, Venners SA, Terwedow HA, Feng Y, Niu T, Li Z, Laird N, Brain JD, Cummings SR, Bouxsein ML, et al: Relation of body composition, fat mass, and serum lipids to osteoporotic fractures and bone mineral density in Chinese men and women. Am J Clin Nutr 83: 146-154, 2006.

24. Zhao LJ, Liu YJ, Liu PY, Hamilton J, Recker RR and Deng HW: Relationship of obesity with osteoporosis. J Clin Endocrinol Metab 92: 1640-1646, 2007.

25. Zhao LJ, Jiang H, Papasian CJ, Maulik D, Drees B, Hamilton J and Deng HW: Correlation of obesity and osteoporosis: Effect of fat mass on the determination of osteoporosis. J Bone Miner Res 23: 17-29, 2008.

26. Matsumoto T, Kano K, Kondo D, Fukuda N, Iribe Y, Tanaka N, Matsubara Y, Sakuma T, Satomi A, Otaki M, et al: Mature adipocyte-derived dedifferentiated fat cells exhibit multilineage potential. J Cell Physiol 215: 210-222, 2008.

27. Schäffler A and Büchler C: Concise review: Adipose tissue-derived stromal cells-basic and clinical implications for novel cell-based therapies. Stem cells 25: 818-827, 2007.

28. Zuk PA, Zhu M, Mizuno H, Huang J, Futrell JW, Katz AJ, Benhaim P, Lorenz HP and Hedrick MH: Multilineage cells from human adipose tissue: Implications for cell-based therapies. Tissue Eng 7: 211-228, 2001.

29. Yoshimura K, Shigeura T, Matsumoto D, Sato T, Takaki Y, Aiba-Kojima E, Sato K, Inoue K, Nagase T, Koshima I and Gonda K: Characterization of freshly isolated and cultured cells derived from the fatty and fluid portions of liposuction aspirates. J Cell Physiol 208: 64-76, 2006. 
30. Kazama T, Fujie M, Endo T and Kano K: Mature adipocyte-derived dedifferentiated fat cells can transdifferentiate into skeletal myocytes in vitro. Biochem Biophys Res Commun 377: 780-785, 2008

31. Oki Y, Watanabe S, Endo T and Kano K: Mature adipocyte-derived dedifferentiated fat cells can trans-differentiate into osteoblasts in vitro and in vivo only by all-trans retinoic acid. Cell Struct Funct 33: 211-222, 2008

32. Jumabay M, Matsumoto T, Yokoyama S, Kano K, Kusumi Y, Masuko T, Mitsumata M, Saito S, Hirayama A, Mugishima H and Fukuda N: Dedifferentiated fat cells convert to cardiomyocyte phenotype and repair infarcted cardiac tissue in rats. J Mol Cell Cardiol 47: 565-575, 2009.

33. Sakuma T, Matsumoto T, Kano K, Fukuda N, Obinata D, Yamaguchi K, Yoshida T, Takahashi S and Mugishima $\mathrm{H}$ : Mature, adipocyte derived, dedifferentiated fat cells can differentiate into smooth muscle-like cells and contribute to bladder tissue regeneration. J Urol 182: 355-365, 2009.
34. Nakamura T, Shinohara Y, Momozaki S, Yoshimoto T and Noguchi K: Co-stimulation with bone morphogenetic protein-9 and FK506 induces remarkable osteoblastic differentiation in rat dedifferentiated fat cells. Biochem Biophys Res Commun 440: 289-294, 2013.

35. Liu HY, Wu AT, Tsai CY, Chou KR, Zeng R, Wang MF, Chang WC, Hwang SM, Su CH and Deng WP: The balance between adipogenesis and osteogenesis in bone regeneration by platelet-rich plasma for age-related osteoporosis. Biomaterials 32: 6773-6780, 2011.

36. Xiong Z, Yan Y, Wang S, Zhang R and Zhang C: Fabrication of porous scaffolds for bone tissue engineering via low-temperature deposition. Scripta Materialia 46: 771-776, 2002. 